\title{
Effects of low-intensity pulsed ultrasound on osteosarcoma and cancer cells
}

\author{
YASUSHI SAWAI $^{1,2}$, HIROAKI MURATA $^{1}$, KAZUTAKA KOTO $^{1,2}$, TAKAAKI MATSUI $^{1}$, NAOYUKI HORIE $^{1}$, \\ EISHI ASHIHARA $^{3}$, TAIRA MAEKAWA ${ }^{3}$, SHINJI FUSHIKI ${ }^{2}$ and TOSHIKAZU KUBO ${ }^{1}$ \\ Departments of ${ }^{1}$ Orthopaedics and ${ }^{2}$ Pathology and Applied Neurobiology, Graduate School of Medical Science, \\ Kyoto Prefectural University of Medicine, 465 Kawaramachi-Hirokoji, Kamigyo-ku, Kyoto 602-8566; \\ ${ }^{3}$ Department of Transfusion Medicine and Cell Therapy, Kyoto University Hospital, \\ 54 Kawahara-cho, Shogoin, Sakyo-ku, Kyoto 606-8507, Japan
}

Received March 14, 2012; Accepted April 26, 2012

DOI: 10.3892/or.2012.1816

\begin{abstract}
Metastatic bone tumors cause pain and pathological fractures due to bone destruction. If we could enhance new osteogenic activities and prevent progression of osteolytic change by malignant cells, patients could achieve satisfactory activity of daily living. Low-intensity pulsed ultrasound (LIPUS), which leads to bone formation by osteoblasts, has been used for the treatment of fractures. LIPUS has been reported to enhance the effect of an anticancer drug on lymphoma and liver cancer cells. However, there have been no reports of proliferation, vascularization and migration effects on cancer cells. In this study, we investigated the effects of LIPUS treatment on cancer and osteosarcoma cells and specifically whether it promotes bone formation without accelerating proliferation of tumor cells. We used MC3T3-E1 cells, a mouse osteoblast cell line, LM8, a mouse osteosarcoma cell line, $\mathrm{SaOS} 2$, a human osteosarcoma cell line, 786-O, a human renal cancer cell line, PC-3, a human prostate cancer cell line, and A549, a human lung cancer cell line. The expression of extracellular signal-regulated kinase (ERK1/2), Akt, $\beta$-catenin, vascular endothelial growth factor (VEGF), and cell migration were analyzed. LIPUS stimulation did not affect proliferation of all the cells examined. The phosphorylation of ERK1/2 and Akt was induced by LIPUS stimulation in MC3T3-E1, LM8, SaOS2 and A549 cells, but not in PC-3 and 786-O cells. LIPUS stimulation did not significantly increase $\beta$-catenin. VEGF protein levels and cell migration were significantly increased only in MC3T3-E1 cells. It may be concluded that LIPUS stimulation on metastatic bone tumors induces differentiation of osteoblasts without prolifera-
\end{abstract}

Correspondence to: Dr Hiroaki Murata, Department of Orthopaedics, Graduate School of Medical Science, Kyoto Prefectural University of Medicine, 465 Kawaramachi-Hirokoji, Kamigyo-ku, Kyoto 602-8566, Japan

E-mail: murah@koto.kpu-m.ac.jp

Key words: low-intensity pulsed ultrasound, metastatic bone tumor, treatment, cancer, osteosarcoma, osteoblast tion of tumor cells. Our study suggests that LIPUS may be a new method of treatment without surgery for metastatic bone tumors.

\section{Introduction}

Many people suffer from metastatic bone tumors caused by a wide variety of cancers. A skeletal-related event (SRE), such as a pathological fracture or spinal cord compression, and the requirement for orthopedic surgery or palliative radiotherapy reduce patients' activity of daily living (ADL) and quality of life (QOL) and worsen prognosis. Treatment for the prevention of an SRE improves patients' ADL and QOL. Osteolytic change with bone metastasis leads to pain and pathological fracture. When surgery, chemotherapy and radiotherapy are not effective, bone destruction progresses. If we could inhibit progression of osteolytic change and enhance osteogenic change more than osteolytic change, it may be possible to prevent fracture and pain and to maintain QOL. In addition, there is a need to develop non-invasive treatment when considering patients' general condition. Low-intensity pulsed ultrasound (LIPUS) stimulation is known as one of the methods for promoting bone formation in orthopedics (1). LIPUS stimulation is an established and widely used intervention for accelerating fracture healing and bone maturation in distraction osteogenesis, and delayed fracture union and nonunion in clinical settings (1-4). To influence bone repair, LIPUS is distinguished by being non-invasive and easy to apply, and the LIPUS signal has a sufficiently low intensity to be considered non-destructive (5). Because tumor cells in metastatic bone tumors coexist with normal osteocytes in impending fractures, osteoblasts and osteoclasts are stimulated by tumor-induced bone destruction all the time. In this condition, we hypothesized that LIPUS stimulates bone formation similar to normal fracture healing and prevents progression of pathological fractures. Effects of LIPUS on cell proliferation, vascularization, and migration in the process of fracture healing have been reported in many cases $(6,7)$. Although the mechanisms involved have not been elucidated, LIPUS stimulation has been reported to affect osteoblast differentiation without increasing cell number of osteoblast $(8,9)$. LIPUS was reported to enhance the effect of an anticancer drug on lymphoma 
and liver cancer cells (10). However, there were no reports of proliferation, vascularization and migration effects on cancer cells. Therefore, there was a need to investigate the effects of LIPUS on cancer cells because LIPUS might stimulate tumor proliferation, vascularization, and migration. In this study, we investigated the effects of LIPUS treatment on cell viability, cell proliferation, vascularization, and migration in mouse osteoblast, mouse and human osteosarcoma, human prostate cancer, renal cancer, and lung cancer cell lines under the same conditions as in clinical use.

\section{Materials and methods}

Cell culture. We used MC3T3-E1, a mouse osteoblast cell line, LM8, a mouse osteosarcoma cell line, SaOS2, a human osteosarcoma cell line, 786-O, a human renal cancer cell line, PC-3, a human prostate cancer cell line, and A549, a human lung cancer cell line. MC3T3-E1 was maintained in alpha-minimum essential medium ( $\alpha$-MEM) with $10 \%$ fetal calf serum (FCS) containing antibiotics (100 U/ml penicillin $\mathrm{G}, 100 \mathrm{mg} / \mathrm{ml}$ streptomycin). LM8, SaOS2, 786-O, and A549 were maintained in Dulbecco's modified Eagle's medium (DMEM) with FCS containing antibiotics. PC-3 was maintained in RPMI-1640 with FCS containing antibiotics. The cells were cultured in a humidified atmosphere of $5 \% \mathrm{CO}_{2}$ and $95 \%$ air at $37^{\circ} \mathrm{C}$.

Ultrasound treatment. An ultrasound exposure system, which was made by Medical Engineering Research Laboratories of Teijin Ltd. (Tokyo, Japan), consisted of an array of six 2.5-cm-diameter PZT-4 (lead-zirconate titanate) transducers, specially designed for a 6-well tissue culture plate. This array was placed at the bottom of a water tank, and the culture plate was located above the array. The temporal average intensity was $30 \mathrm{~mW} / \mathrm{cm}^{2}$ and the frequency was $1.5 \mathrm{MHz}$ with a $200-\mu \mathrm{s}$ tone burst repeated at $1.0 \mathrm{KHz}(11,12)$. This setting is the same as in clinical use. LIPUS was administered for 20 min every day for the duration of this experiment.

Determination of cell number. MC3T3-E1 cells were seeded in a 6-well plate at a density of $2.0 \times 10^{4}$ cells $/ \mathrm{cm}^{2}$. LM8, SaOS2, PC-3, 786-O, and A549 cells were seeded at a density of $1.0 \times 10^{4} \mathrm{cells} / \mathrm{cm}^{2}$. The cells were cultured in the presence or absence of daily LIPUS stimulation for up to $72 \mathrm{~h}$. The cells were detached by gentle trypsinization and counted microscopically using a trypan blue dye exclusion test.

Western blot analysis. MC3T3-E1, LM8, SaOS2, PC-3, 786-O, and A549 cells were plated in a 6-well tissue cell culture plate at a density of $5.0 \times 10^{4} \mathrm{cells} / \mathrm{cm}^{2}$ and were harvested 5, $30 \mathrm{~min}, 1,3,6,12,24$, and $48 \mathrm{~h}$ after LIPUS stimulation. The cells were washed twice with PBS and lysed with RIPA buffer [20 mM Tris-HCl (pH 7.4), $150 \mathrm{mM} \mathrm{NaCl}, 0.1 \%$ SDS, $1 \%$ Nonidet P-40, $0.5 \%$ sodium deoxycholate, $40 \mathrm{mM} \mathrm{NaF}$, and protease inhibitor cocktail (Sigma, St. Louis, MO, USA)]. The lysates were centrifuged at $15,000 \mathrm{rpm}$ for $20 \mathrm{~min}$. The supernatant lysate with sample buffer [0.0625 M Tris-HCl (pH 6.8), $2 \%$ SDS, $5 \%$ glycerol, $5 \%$ 2-ME] was boiled at $95^{\circ} \mathrm{C}$ for $5 \mathrm{~min}$. The samples were separated on sodium dodecyl sulfate-polyacrylamide gel electrophoresis (SDS-PAGE) and then electroblotted onto a nitrocellulose membrane (Amersham
Biosciences, Tokyo, Japan). The membranes were saturated with 5\% (wt/vol) non-fat dry milk in Tris-buffered saline with Tween-20 (TBST) [25 mM Tris- $\mathrm{HCl}$ (pH 7.8), $140 \mathrm{mM} \mathrm{NaCl}$, $0.1 \%$ (vol/vol) Tween-20] and then incubated overnight with the following antibodies (diluted 1:1,000 in TBST): extracellular signal-regulated kinase (ERK1/2) (Cell Signaling Technology, Beverly, MA, USA), phosphorylated ERK1/2 (pERK1/2) (Cell Signaling Technology), Akt (Cell Signaling Technology), phosphorylated Akt (p-Akt) (Cell Signaling Technology) and $\beta$-catenin (Becton-Dickinson, Franklin Lakes, NJ, USA). The membranes were washed thoroughly with TBST and incubated for $1 \mathrm{~h}$ with horseradish peroxidase-conjugated anti-mouse or -rabbit IgG (Santa Cruz Biotechnologies, Santa Cruz, CA, USA) (diluted 1:5,000 in TBST). Detection was performed with enhanced chemiluminescence kits (Amersham Biosciences).

Enzyme-linked immunosorbent assay (ELISA) analysis. MC3T3-E1, LM8, SaOS2, PC-3, 786-O, and A549 cells were plated in a 6-well tissue cell culture plate at a density of $5.0 \times 10^{4}$ cells $/ \mathrm{cm}^{2}$. After $48 \mathrm{~h}$, each culture medium was exchanged with $1 \mathrm{ml}$ of serum-free medium. Twenty-four hours after the medium exchange, the cells were treated with LIPUS. The supernatants were harvested $0,3,6,12,24$, and $48 \mathrm{~h}$ after LIPUS stimulation. ELISA assays for vascular endothelial growth factor (VEGF) were performed with the human and mouse VEGF ELISA kit (Ray Biotech, Norcross, GA, USA).

Wound healing assay. MC3T3-E1, LM8, PC-3, SaOS2, 786-O, and A549 cells were cultured until confluence. The confluent cell monolayer in a 6-well plate was wounded by manually scraping the cells with a pipette tip. The cells were treated with or without LIPUS stimulation. Cell migration into the wound surface was monitored by microscopy at 0,6 , and $12 \mathrm{~h}$ after LIPUS stimulation. Quantitation was carried out in terms of $\%$ wounded area filled [\% wounded area filled $=100 \times$ (initial width of wounding - final width)/initial] by measuring the distance of the wound edge of the migrating cells from the start point to the migrated point from 3 independent experiments (13).

Statistical analysis. The data are represented as the mean \pm standard deviation (SD). Statistical significance was determined using Student's t-test. $\mathrm{P}<0.05$ was considered statistically significant.

\section{Results}

Cell number. The cell number did not change regardless of the presence or absence of daily LIPUS stimulation, up to 3 days (Fig. 1).

Phosphorylation of ERK and Akt and expression of $\beta$-catenin. ERK1/2: immunoreactive bands at $44 \mathrm{kDa}$ (ERK1 and phosphorylated ERK1 protein) and $42 \mathrm{kDa}$ (ERK2 and phosphorylated ERK2 protein) were observed. LIPUS stimulation on MC3T3-E1 and LM8 temporarily induced phosphorylation of ERK1/2 between 5 min and $1 \mathrm{~h}$ after LIPUS stimulation. LIPUS stimulation on SaOS2 and A549 bimodally induced phosphorylation of ERK1/2 at 1 and $24 \mathrm{~h}$. LIPUS stimulation on PC-3 and 786-O did not induce phosphorylation of ERK1/2 (Fig. 2A). 

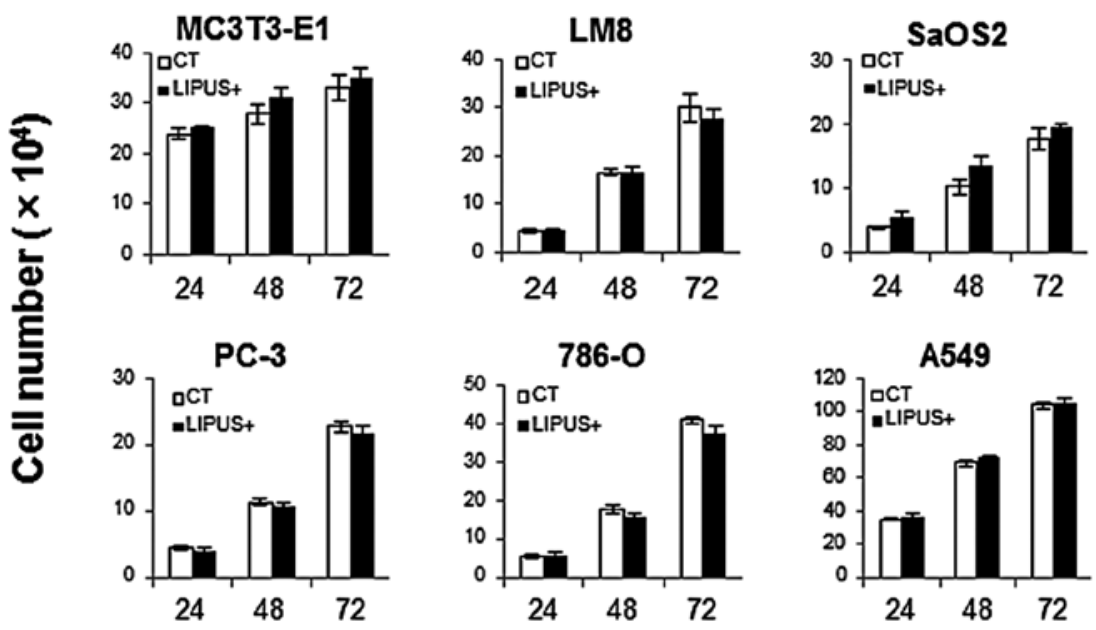

\section{Incubation time (h)}

Figure 1. LIPUS did not affect the cell number. Each cell type was cultured in the presence (black bars, LIPUS+) or absence (white bars, CT) of daily LIPUS stimulation for up to $72 \mathrm{~h}$. The cell number was compared with control culture by counting the cells using a trypan blue dye exclusion test. The rate of increase in the cell numbers showed no significant difference. The data are shown as the mean $\pm \mathrm{SD}$ (bars, $\pm \mathrm{SD}$ ) of at least three independent experiments.

A Phosphorylation of ERK1/2

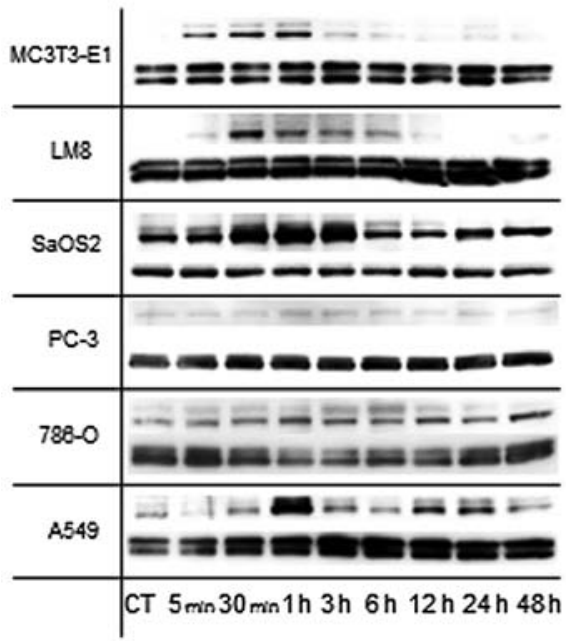

B Phosphorylation of Akt1/2

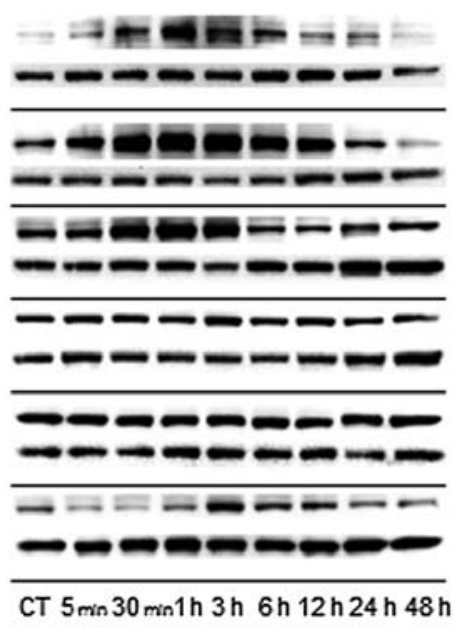

C $\beta$-catenin

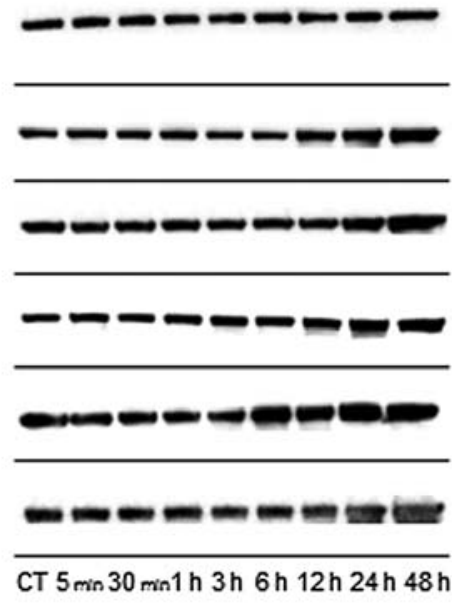

Figure 2. LIPUS stimulation on MC3T3-E1, LM8, SaOS2, and A549 induced phosphorylation of ERK1/2 and Akt. Each cell type was harvested between 5 min and $48 \mathrm{~h}$ after LIPUS stimulation. The expressions of pERK1/2, ERK1/2, pAkt, Akt, and $\beta$-catenin were analyzed by western blotting. (A) The expressions of ERK1/2 and pERK1/2 were observed (upper, pERK; lower, ERK). Phosphorylation of ERK1/2 on MC3T3-E1, LM8, SaOS2, and A549 was induced by LIPUS stimulation, but not in PC-3 and 786-O. (B) The expressions of Akt and pAkt were observed (upper, pAkt; lower, Akt). Phosphorylations of Akt on MC3T3-E1, LM8, SaOS2, and A549 were induced by LIPUS stimulation, but not in PC-3 and 786-O. (C) The expression of $\beta$-catenin was observed. $\beta$-catenin expression was not increased by LIPUS stimulation.

Akt: immunoreactive bands at $60 \mathrm{kDa}$ (Akt and phosphorylated Akt protein) were observed. LIPUS stimulation on MC3T3-E1 temporarily induced phosphorylation of Akt between $30 \mathrm{~min}$ and $24 \mathrm{~h}$. LIPUS stimulation on LM8 temporarily induced phosphorylation of Akt between $5 \mathrm{~min}$ and $12 \mathrm{~h}$. LIPUS stimulation on SaOS2 temporarily induced phosphorylation of Akt between $30 \mathrm{~min}$ and $3 \mathrm{~h}$. LIPUS stimulation on A549 temporarily induced phosphorylation of Akt at $3 \mathrm{~h}$. LIPUS stimulation on PC-3 and 786-O did not induce phosphorylation of Akt (Fig. 2B). $\beta$-catenin: immunoreactive bands at $92 \mathrm{kDa}(\beta$-catenin protein) were observed. LIPUS stimulation of the cells did not significantly increase $\beta$-catenin expression (Fig. 2C).

Expression of VEGF protein. VEGF protein levels were assessed in the collected culture media by sandwich-ELISA. VEGF protein levels in MC3T3-E1 at 12, 24, and $48 \mathrm{~h}$ after LIPUS stimulation were significantly increased compared with those of the control, whereas those in LM8, SaOS2, PC-3, 786-O, and A549 were not (Fig. 3). 


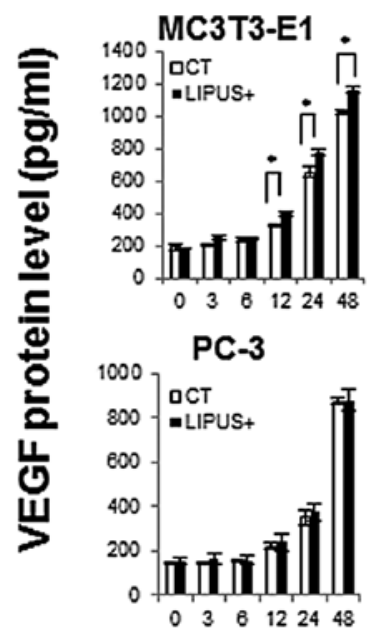

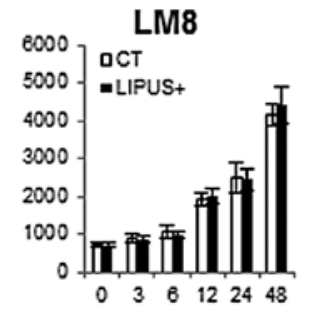

$786-0$

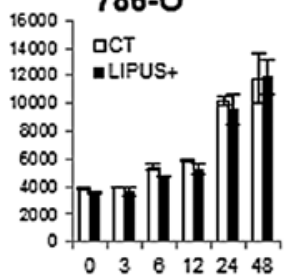

Incubation time $(\mathrm{h})$
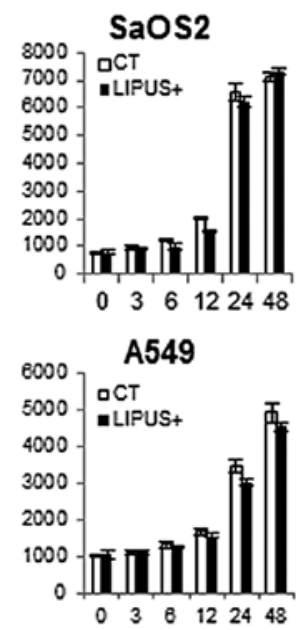

${ }^{*} \mathrm{p}<0.05$

Figure 3. LIPUS stimulation increased VEGF protein levels only in MC3T3-E1. Each cell type was cultured in the presence (black bars, LIPUS+) or absence (white bars, CT) of daily LIPUS stimulation for up to $48 \mathrm{~h}$. The supernatants of MC3T3-E1, PC-3, LM8, SaOS2, 786-O, and A549 cells were harvested between 0, 3, 6, 12, 24, and $48 \mathrm{~h}$ after LIPUS stimulation. VEGF protein levels were assessed in the collected culture media by sandwich-ELISA. VEGF protein levels in MC3T3-E1 were significantly increased compared with those of the control and not significantly increased in LM8, SaOS2, PC-3, 786-O, and A549 ("p<0.05). The data are shown as the mean $\pm \mathrm{SD}$ (bars, $\mathrm{SD}$ ) of at least three independent experiments.

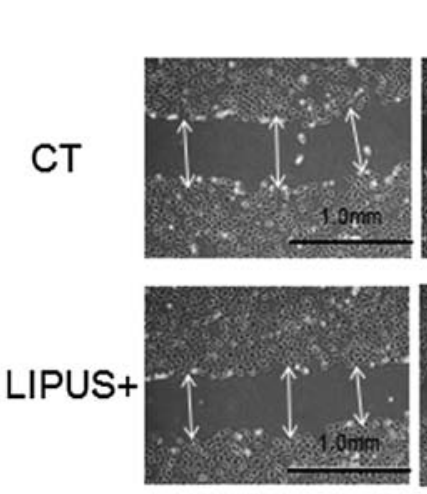

$\mathrm{Oh}$

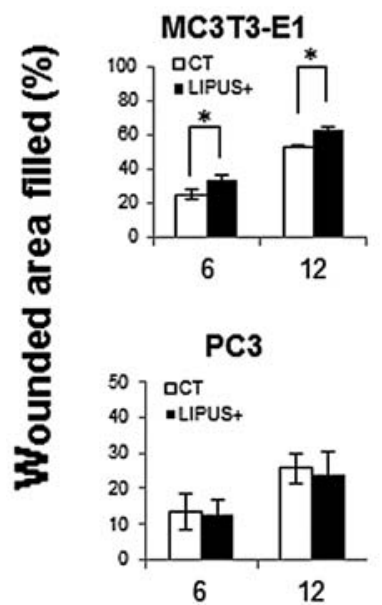

\section{MC3T3-E1}
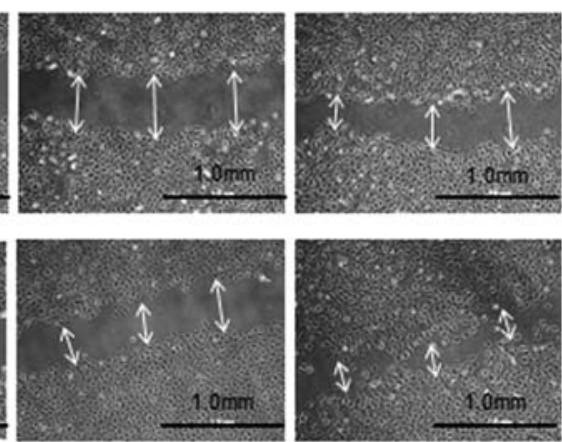

$6 \mathrm{~h}$

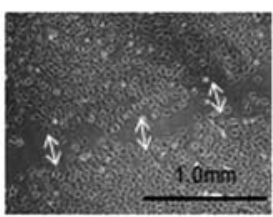

$12 \mathrm{~h}$

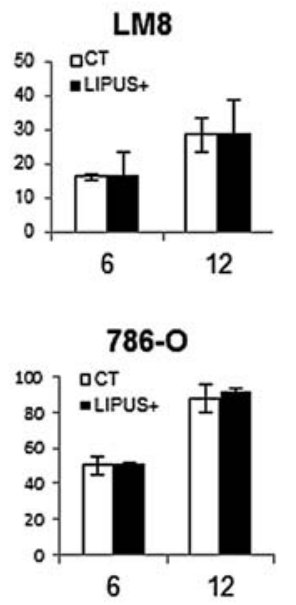

6

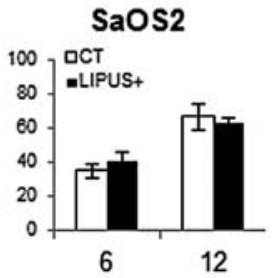

A549

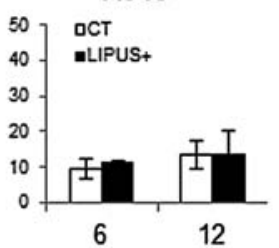

\section{Incubation time (h)}

Figure 4. LIPUS stimulation promoted cell migration only in MC3T3-E1. Each cell type was cultured in the presence (black bars, LIPUS+) or absence (white bars, CT) of daily LIPUS stimulation for up to $12 \mathrm{~h}$. The cell migration of MC3T3-E1, LM8, SaOS2, PC-3, 786-O, and A549 cells into the wound surface was monitored by microscopy at 0,6 , and $12 \mathrm{~h}$ after LIPUS stimulation. Quantitation was carried out in terms of $\%$ wounded area filled [\% wounded area filled $=100 \mathrm{x}$ (initial width of wounding - final width)/initial] measuring the distance of the wound edge of the migrating cells from the start point to the migrated point. LIPUS stimulation resulted in significant promotion of MC3T3-E1 cell migration. SaOS2, PC-3, 786-O, and A549 cells were not affected ("p<0.05). The data are shown as the mean \pm SD (bars, \pm SD) of at least three independent experiments. 
Cell migration. In order to investigate the effect of LIPUS stimulation on cell migration, scratch wound healing assays were performed. LIPUS stimulation resulted in significant promotion of MC3T3-E1 cell migration. MC3T3-E1 cell migration increased by 8.7 and $9.4 \%$ at 6 and $12 \mathrm{~h}$, respectively, compared with those of control cells. LM8, SaOS2, PC-3, 786-O, and A549 cells were not affected (Fig. 4).

\section{Discussion}

Application of adequate mechanical stress to bone is essential for maintaining bone mass and strength. Various types of mechanical loading have been clinically tested for their bone mass-promoting activity in the treatment of bone fractures. Among them, LIPUS was reported to promote healing of bone fracture in 1983 (1). Then it was widely reported that LIPUS reduced the time required for fracture healing $(14,15)$. Furthermore, LIPUS induced bone maturation in distraction osteogenesis, and delayed fracture union and nonunion in animal models as well as in clinical settings (1-4). In vitro experiments have demonstrated that LIPUS stimulation affects mouse and rat osteoblast differentiation without influencing proliferation $(8,9)$. Although the mechanisms involved have not been elucidated, LIPUS transmits signals into the cell via an integrin that acts as a mechanoreceptor on the cell membrane (16). The integrin/Ras/MAPK pathway is known to be a general pathway involved in cell proliferation. A previous study demonstrated that ERK phosphorylation increased after LIPUS stimulation on MC3T3-E1, starting at $5 \mathrm{~min}$, reaching a maximum between 15 and $30 \mathrm{~min}$, and then gradually decreasing, and that LIPUS stimulation on human pre-osteoblastic cells progressively increased ERK phosphorylation in $1 \mathrm{~h}(17,18)$. The PI3K/Akt pathway, on the other hand, is known to be involved in various functions such as cell survival, proliferation, motility, control of cell size, and metabolism. It was reported that LIPUS exposure in MC3T3-E1 increased Akt phosphorylation in a time-dependent manner and maximal activation was detected $15 \mathrm{~min}$ after LIPUS stimulation (17). In the present study, LIPUS stimulation on MC3T3-E1 temporarily induced phosphorylation of ERK1/2 between $5 \mathrm{~min}$ and $1 \mathrm{~h}$ and phosphorylation of Akt between $30 \mathrm{~min}$ and $24 \mathrm{~h}$ after LIPUS stimulation. These results are comparable to those of a previous study.

$\mathrm{Wnt} / \beta$-catenin signaling pathway has been reported to play a crucial role in cell proliferation, differentiation, and possibly apoptosis (19). There have been no reports of studies investigating the effect of LIPUS stimulation on $\beta$-catenin expression. Instead, mechanical loading in mouse tibial bone increased expression of canonical Wnt pathway and Wnt/ $\beta$-catenin target genes (20). Our experiment indicated that LIPUS stimulation did not significantly increase $\beta$-catenin expression.

VEGF has a central role in the regulation of vascularization. VEGF expression has been reported to be increased by LIPUS stimulation in human fetal pre-osteoblastic cell and rabbit bone-tendon junction $(6,21)$. Migration of osteoblasts has an important impact on fracture healing. Our experiment indicated that LIPUS stimulation of osteoblasts, MC3T3-E1, promoted cell migration. MC3T3-E1 cell migration increased by 8.7 and $9.4 \%$ at 6 and $12 \mathrm{~h}$, respectively, compared with that of control cells. In addition, VEGF protein levels in MC3T3-E1 at 12, 24, and $48 \mathrm{~h}$ after LIPUS stimulation were significantly increased compared with those of the control. These changes may help differentiation and formation of bone. However, for malignant cells, these changes observed in osteoblastic cells may favor tumor growth. Therefore, we next studied whether LIPUS also affects malignant cells, viz., osteosarcoma cells, and other visceral cancer cells.

Osteosarcoma is generally characterized by exhibition of osteoblastic differentiation and production of osteoid matrix. LIPUS effects on osteosarcoma cell lines such as an osteoblastic cell line has been reported $(9,22)$. In the present study, LIPUS on LM8 and SaOS2 induced phosphorylation of ERK1/2 and Akt and did not affect cell number, $\beta$-catenin expression, VEGF protein expression, or cell migration. Although phosphorylated ERK and Akt in osteosarcoma were generally considered to activate the tumor cell proliferation, Cagnol and Chambard reported that activation of ERK1/2 in osteosarcoma cells induced apoptosis and autophagy (23). From our study, it was concluded that LIPUS stimulation did not affect the cell number of osteosarcoma. Moreover, angiogenic and migration effects were different from those of osteoblast cells. It is tempting to speculate that LIPUS stimulation of osteosarcoma cells might promote bone differentiation and reduce the activity of a tumor, although our results are limited.

Two types of cancer cells, 786-O and PC-3, were not affected by LIPUS stimulation in any of the experiments. It may thus be argued that LIPUS used for bone metastases from renal and prostate cancer induces osteoblastic differentiation without inducing cancer proliferation, vascularization, and migration. On the other hand, the effect of LIPUS stimulation of A549 induced phosphorylation of ERK1/2 and Akt and did not affect cell number, $\beta$-catenin expression, VEGF protein expression, or cell migration. Although activations of ERK and Akt were considered to induce proliferation of lung cancer cells, it was reported that activation of ERK is required for apoptosis in A549 lung cancer cells $(24,25)$. In our study, LIPUS stimulation did not enhance proliferative activity of cancer cells, regardless of the tissue of origin.

Generally, a part of intact bone is substituted with malignant cells in metastatic bone tumor and malignant bone tumor. Progression of tumor growth causes pain and bone destruction, resulting in pathological bone fracture. Tumor growth stimulates activation of osteoclasts leading to bone resorption whereby more space is produced adequate for malignant cell proliferation. Stimulation to osteoclasts essentially occurs in combination with stimulation of osteoblasts. Metastatic bone tumor is similar to bone fracture because both osteoclasts and osteoblasts are stimulated. However, proliferative activity of malignant cells is generally greater than that of osteoblasts. If we could enhance new osteogenic activities in spite of malignant cell growth, it would stop progression of the osteolytic change which may prevent bone fracture. Patients could thus achieve relief of pain, prevention of SRE, and finally satisfactory ADL and QOL.

This study model showed limited outcome because in vitro experiments are different from in vivo environments of metastatic bone tumor. The mechanisms by which metastases are formed are complex, involving many types of cells and steps that include angiogenesis, invasion, and proliferation in the bone microenvironment. Tumor cells in the bone micro- 
environment produce a large number of cytokines such as tumor-produced parathyroid hormone-related protein (PTHrP), transforming growth factor $\beta$, and interleukin-6 (26).

In conclusion, LIPUS did not significantly increase cellular proliferation, migration or VEGF production of malignant cells compared with that without LIPUS. In contrast, LIPUS induced migration and VEGF production without proliferation in osteoblasts. LIPUS when applied on metastatic bone tumors might be beneficial by inducing osteoblast differentiation without cancer proliferation. In the future, LIPUS stimulation might be one of the treatments of metastatic bone tumor because of its noninvasiveness.

\section{References}

1. Duarte LR: The stimulation of bone growth by ultrasound. Arch Orthop Trauma Surg 101: 153-159, 1983.

2. Heckman JD, Ryaby JP, McCabe J, Frey JJ and Kilcoyne RF: Acceleration of tibial fracture-healing by non-invasive, lowintensity pulsed ultrasound. J Bone Joint Surg Am 76: 26-34, 1994.

3. Rutten S, Nolte PA, Guit GL, Bouman DE and Albers GH: Use of low-intensity pulsed ultrasound for posttraumatic nonunions of the tibia: a review of patients treated in the Netherlands. J Trauma 62: 902-908, 2007.

4. Wang SJ, Lewallen DG, Bolander ME, Chao EY, Ilstrup DM and Greenleaf JF: Low intensity ultrasound treatment increases strength in a rat femoral fracture model. J Orthop Res 12: 40-47, 1994.

5. Dimitriou R and Babis GC: Biomaterial osseointegration enhancement with biophysical stimulation. J Musculoskelet Neuronal Interact 7: 253-265, 2007.

6. Wang FS, Kuo YR, Wang CJ, et al: Nitric oxide mediates ultrasound-induced hypoxia-inducible factor-1alpha activation and vascular endothelial growth factor-A expression in human osteoblasts. Bone 35: 114-123, 2004.

7. Tang CH, Lu DY, Tan TW, Fu WM and Yang RS: Ultrasound induces hypoxia-inducible factor-1 activation and inducible nitric-oxide synthase expression through the integrin/integrinlinked kinase/Akt/mammalian target of rapamycin pathway in osteoblasts. J Biol Chem 282: 25406-25415, 2007.

8. Bandow K, Nishikawa Y, Ohnishi T, et al: Low-intensity pulsed ultrasound (LIPUS) induces RANKL, MCP-1, and MIP-1beta expression in osteoblasts through the angiotensin II type 1 receptor. J Cell Physiol 211: 392-398, 2007.

9. Suzuki A, Takayama T, Suzuki N, Sato M, Fukuda T and Ito K: Daily low-intensity pulsed ultrasound-mediated osteogenic differentiation in rat osteoblasts. Acta Biochim Biophys Sin 41: 108-115, 2009.

10. Kondo T, Yoshida T, Ogawa R, et al: Low-intensity ultrasound adjuvant therapy: enhancement of doxorubicin induced cytotoxicity and the acoustic mechanisms involved. J Med Ultrasonics 36: 61-68, 2009.

11. Ito M, Azuma Y, Ohta T and Komoriya K: Effects of ultrasound and 1,25-dihydroxyvitamin D3 on growth factor secretion in co-cultures of osteoblasts and endothelial cells. Ultrasound Med Biol 26: 161-166, 2000.
12. Iwabuchi S, Ito M, Hata J, Chikanishi T, Azuma Y and Haro H: In vitro evaluation of low-intensity pulsed ultrasound in herniated disc resorption. Biomaterials 26: 7104-7114, 2005.

13. Tano K, Mizuno R, Okada T, et al: MALAT-1 enhances cell motility of lung adenocarcinoma cells by influencing the expression of motility-related genes. FEBS Lett 584: 4575-4580, 2010.

14. Dyson M and Brookes M: Stimulation of bone repair by ultrasound. Ultrasound Med Biol 2: 61-66, 1983.

15. Kristiansen TK, Ryaby JP, McCabe J, Frey JJ and Roe LR: Accelerated healing of distal radial fractures with the use of specific, low-intensity ultrasound. A multicenter, prospective, randomized, double-blind, placebo-controlled study. J Bone Joint Surg Am 79: 961-973, 1997.

16. Takeuchi R, Ryo A, Komitsu N, et al: Low-intensity pulsed ultrasound activates the phosphatidylinositol 3 kinase/Akt pathway and stimulates the growth of chondrocytes in three-dimensional cultures: a basic science study. Arthritis Res Ther 10: R77, 2008.

17. Tang CH, Yang RS, Huang TH, Lu DY, Chuang WJ, Huang TF and Fu WM: Ultrasound stimulates cyclooxygenase-2 expression and increases bone formation through integrin, focal adhesion kinase, phosphatidylinositol 3-kinase, and Akt pathway in osteoblasts. Mol Pharmacol 69: 2047-2057, 2006.

18. Chen YJ, Wang CJ, Yang KD, et al: Pertussis toxin-sensitive Galphai protein and ERK-dependent pathways mediate ultrasound promotion of osteogenic transcription in human osteoblasts. FEBS Lett 554: 154-158, 2003.

19. Almeida M, Han L, Bellido T, Manolagas SC and Kousteni S: Wnt proteins prevent apoptosis of both uncommitted osteoblast progenitors and differentiated osteoblasts by beta-catenindependent and -independent signaling cascades involving $\mathrm{Src} /$ ERK and phosphatidylinositol 3-kinase/AKT. J Biol Chem 280: 41342-41351, 2005.

20. Robinson JA, Chatterjee-Kishore M, Yaworsky PJ, et al: Wnt/betacatenin signaling is a normal physiological response to mechanical loading in bone. J Biol Chem 281: 31720-31728, 2006.

21. Lu H, Qin L, Cheung W, Lee K, Wong W and Leung K: Low-intensity pulsed ultrasound accelerated bone-tendon junction healing through regulation of vascular endothelial growth factor expression and cartilage formation. Ultrasound Med Biol 34: 1248-1260, 2008

22. Borsje MA, Ren Y, de Haan-Visser HW and Kuijer R: Comparison of low-intensity pulsed ultrasound and pulsed electromagnetic field treatments on OPG and RANKL expression in human osteoblastlike cells. Angle Orthod 80: 498-503, 2010.

23. Cagnol S and Chambard JC: ERK and cell death: mechanisms of ERK-induced cell death-apoptosis, autophagy and senescence. FEBS J 277: 2-21, 2010

24. Nguyen TT, Tran E, Nguyen TH, Do PT, Huynh TH and Huynh H: The role of activated MEK-ERK pathway in quercetin-induced growth inhibition and apoptosis in A549 lung cancer cells. Carcinogenesis 25: 647-659, 2004.

25. Jin CY, Moon DO, Lee JD, et al: Sulforaphane sensitizes tumor necrosis factor-related apoptosis-inducing ligand-mediated apoptosis through downregulation of ERK and Akt in lung adenocarcinoma A549 cells. Carcinogenesis 28: 1058-1066, 2007.

26. Guise TA: Molecular mechanisms of osteolytic bone metastases. Cancer 88: 2892-2898, 2000. 